\title{
A CASE STUDY FOR MODELLING CANCER INCIDENCE USING BAYESIAN SPATIO-TEMPORAL MODELS
}

\author{
Su Yun KAnG ${ }^{1,2 *}$, JAMEs MCGReE ${ }^{1,2}$, Peter BAAdE ${ }^{3,4,5}$ KeRrie $^{2}$ \\ MENGERSEN $^{1,2}$
}

\begin{abstract}
Summary
Researchers familiar with spatial models are aware of the challenge of choosing the level of spatial aggregation. Few studies have been published on the investigation of temporal aggregation and its impact on inferences regarding disease outcome in space-time analyses. We perform a case study for modelling individual disease outcomes using several Bayesian hierarchical spatio-temporal models, while taking into account the possible impact of spatial and temporal aggregation. Using longitudinal breast cancer data in South East Queensland, Australia, we consider both parametric and non-parametric formulations for temporal effects at various levels of aggregation. Two temporal smoothness priors are considered separately, where each is modelled with fixed effects for the covariates and an intrinsic conditional autoregressive prior for the spatial random effects. Our case study has revealed that different model formulations produce considerably different model performances. For this particular dataset, a classical parametric formulation that assumes a linear time trend produces the best fit among the five models considered. Different aggregation levels of temporal random effects were found to have little impact on model goodness-of-fit and estimation of fixed effects.
\end{abstract}

Key words: Bayesian modelling; integrated nested Laplace approximation; spatial epidemiology; spatio-temporal; temporal aggregation

\section{Introduction}

Epidemiological data are often characterized by a spatial and/or temporal structure. In order to better understand the evolution of a disease over time, it is essential to study space-time variation of a disease. Historically, the common approach in disease mapping is the aggregation of disease data over space and time to match the levels of spatial and

\footnotetext{
* Author to whom correspondence should be addressed.

*E-mail: s7.kang@qut.edu.au ${ }^{1}$ Mathematical Sciences School, Queensland University of Technology, GPO Box 2434, Brisbane QLD 4001, Australia. ${ }^{2}$ CRC for Spatial Information, 204 Lygon Street, Carlton, Victoria 3053, Australia. ${ }^{3}$ Viertel Centre for Research in Cancer Control, Cancer Council Queensland, Gregory Tce, Fortitude Valley, Australia. ${ }^{4}$ School of Public Health, Queensland University of Technology, GPO Box 2434, Brisbane QLD 4001, Australia. ${ }^{5}$ Griffith Health Institute, Griffith University, Australia.

Acknowledgments. The work was supported by the Cooperative Research Centre for Spatial Information, whose activities are funded by the Australian Commonwealth's Cooperative Research Centres Programme. Peter Baade is supported by an NHMRC Career Development Fellowship (\#1005334). The authors would like to thank Cancer Council Queensland for supplying the data for analysis, Susanna Cramb for assisting with the data and two anonymous reviewers for their helpful comments.
} 
temporal aggregation of census data. Despite the possible loss of information resulting from this practice, the aggregation in time and/or space (e.g. 308 Statistical Local Areas for the present study) is required in some instances to see non-zero prevalence (Lawson 2013).

Extension of hierarchical spatial models to include a time dimension has been explored in the last two decades (Bernardinelli, Clayton, Pascutto et al. 1995; Waller, Carlin, Xia et al. 1997; Knorr-Held \& Besag 1998; Knorr-Held 2000; Nobre, Schmidt \& Lopes 2005; Richardson, Abellan \& Best 2006; MacNab \& Gustafson 2007; Abellan, Richardson \& Best 2008). These studies have proposed a range of space-time formulations and demonstrated spatio-temporal analyses of the variation of disease risk. This literature has become the foundation on which subsequent space-time analyses are based; see, for example, Ugarte, Goicoa, Ibáñez et al. (2009). Among other approaches, Bayesian hierarchical models have been widely employed in disease mapping due to their flexibility and ability to accommodate unobserved quantities from a number of discrete levels (Richardson \& Best 2003).

In practice, the choice of spatial aggregation level usually follows that defined by the administrative districts or census regions, whereas the choice of temporal aggregation level is somewhat arbitrary and dependent on the time interval of the reported risk factors. The aggregation of disease cases over a period of years is often motivated by the belief that this will stabilize the rates, given the higher numerators and denominators (Ocaña-Riola 2010). However, studies have shown that aggregation of long time periods may give rise to a bias in estimates of disease rates and relative risks (Ocaña-Riola 2007; Abellan, Richardson \& Best 2008). Despite the required attention and care on the levels of time aggregation, limited studies have been published on the investigation of temporal aggregation and the impact on inferences regarding disease outcome in space-time analyses.

This study aims to assess the suitability of a range of Bayesian spatio-temporal models for our case study, while taking the possible impact of spatial and temporal aggregation into consideration. This is achieved by considering six temporal aggregation levels as well as two levels of spatial aggregation. Leyk, McCormick \& Nuckols (2011) investigate the impact of changing temporal aggregation on spatial distribution of pediatric diarrhea mortality patterns and their associations with risk factors. However, our ultimate objective is to identify a spatio-temporal model that best explains our data, while considering the effects of spatial and temporal aggregation.

To motivate our research, we investigate the relationship between area-disadvantage and individual characteristics, and the risk of being diagnosed as having advanced breast cancer, using longitudinal breast cancer data in South East Queensland, Australia. Using information on the different stages of cancer incidence at time of diagnosis, Knorr-Held, Raßer \& Becker (2002) discover large spatial differences in the stage proportions. In addition to a spatial 
effect, we incorporate a time dimension by either modelling the temporal variation parametrically or non-parametrically.

The paper is organized as follows. In Section 2, we provide details about the case study data. Section 3 describes the methods including model, computation, priors, and model selection criteria. The results of model fitting are reported in Section 4. Section 5 discusses some of the issues and provides some overall conclusions. Throughout the paper, we have used 'aggregation level' to denote different levels of discretisation of the spatio-temporal random effects. To avoid confusion, we have refrained from using the word 'scale' here as it is usually associated with a continuous quantity.

\section{Case study data}

Ethics and Data Custodian approval for this research was obtained from Queensland Health (HREC/09/QHC/25). The breast cancer data were extracted from the populationbased Queensland Cancer Registry (QCR). The data consist of all women diagnosed as having invasive breast cancer in Queensland between 1 January 1996 and 31 December 2009 (inclusive). We focus on breast cancer cases in a study region in south-east Queensland with latitude ranging from -28 to -27.29315 and longitude ranging from 151.91364 to 153.18721 (see Figure 1). The study region extends from Brisbane (on the upper right), the capital and most populous city in Queensland, to Toowoomba (on the middle left), the most populous non-capital inland city in Queensland. A total of 12443 cancer cases were included in the study, with 2121 advanced breast cancer cases and 10322 non-advanced breast cancer cases. We follow the definition of advanced breast cancer in Baade, Turrell \& Aitken (2011). As shown in Figure 1, the cancer cases are spatially sparse with a mixture of dense and scarce points across the study region.

We obtained data for each case on individual-level characteristics (i.e., age, occupation, marital status, indigenous status, and exact date of diagnosis) as well as measures of geographic remoteness and area disadvantage defined at the census region level, namely Statistical Local Areas (SLAs). With respect to the area level covariates, since the information was only obtained at the SLA level, we thus assigned the scores to the patients based on the SLAs in which they resided.

[Figure 1 about here.]

\section{Patients' characteristics of interest at time of diagnosis}

1. Age group (AGE) - Age at diagnosis was collapsed into five-year age groups from $<30$, $30-34, \ldots, 75-79$ and $80+$ years, resulting in twelve age groups. 
2. Occupation (OCCUP) - Five categories of occupation are blue collar (including tradespersons, plant and machine operators and drivers, and labourers and related workers), white collar (including clerks, salespersons and personal service workers), professional (including managers, administrators, professionals and para-professional), not in workforce (including retired, students, unemployed and home duties) and unknown (no information available).

3. Marital status (MARITAL) — Women's marital status includes single, married, widowed, divorced, separated or not stated.

4. Indigenous status (INDIG) - Women's indigenous status includes non-indigenous, indigenous or not stated.

5. Geographic remoteness (ARIA) - ARIA+ classification (AIHW 2004) was used to categorize the remoteness of residence when diagnosed as having breast cancer. This purely geographical measure of remoteness classifies residence into five categories including Major City, Inner Regional, Outer Regional, Remote, and Very Remote areas based on road distance from a locality to the closest service centre in each of five classes of population size.

6. Index of relative socio-economic disadvantage (IRSD) - Socio-economic disadvantage of the SLAs was measured using the Index of Relative Socio-economic Disadvantage (IRSD) calculated by Australian Bureau of Statistics (Adhikari 2006). The IRSD measures disadvantage using factors such as the percentage of residents in each SLA with low income, low educational attainment, high unemployment and jobs in relatively unskilled occupations. The IRSD was collapsed into five quintiles, with Quintile 5 being least disadvantaged and Quintile 1 being most disadvantaged.

\section{Spatial aggregation}

Each cancer case also contains the longitude and latitude co-ordinates of the patient's residential address as well as the SLA variable, which denotes the spatial entity where a patient resides. The co-ordinate information was used to assign patients to their respective grid cells after partitioning the study region into regular grid cells. To model the spatial random effects, we consider the partitions at the SLA level and the grid level by discretizing the study region using grid $30 \times 30$ resulting in 900 grid cells. The choice of the grid $30 \times 30$ is motivated by the findings in Kang, McGree, Baade et al. (2014) where this geographical scale appeared to be a reasonable choice to model the spatial random effects. The adjacency matrix for the SLAs is calculated using the program GeoDa (Anselin, Syabri \& Kho 2006) using first order queen definition of adjacency, where the SLAs are considered to be neighbours if they share a common border or vertex. On the other hand, the cell $2 \mathrm{nb}$ function in the spdep R package (Bivand, Anselin, Berke et al. 2011) is used to generate a list of neighbours for the grid cells, by applying a queen definition of neighbourhood, where 
two grid cells are termed neighbours if they share a common edge or vertex.

\section{Temporal aggregation}

Information on time of diagnosis of each patient is used to construct various levels of temporal aggregation which are used to model temporal random effects. The time of diagnosis (January 1996 to December 2009) was aggregated into various aggregation levels, including 3-year, 2.5-year, 2-year, 1.5-year, 1-year or 0.5-year. These aggregation levels were chosen so as to explore the impact of temporal aggregation.

\section{Methods}

We extend the study of spatial aggregation undertaken by Kang, McGree, Baade et al. (2014) on the same data via the inclusion of a time component into the described spatial model to form a Bayesian spatio-temporal model to estimate the individual disease risk. In addition to spatial random effects, temporal random effects and space-time interaction effects are also considered. As described above, two levels of spatial aggregation are chosen and six levels for temporal aggregation are selected. The model outcomes are compared across different models at different spatio-temporal aggregation levels.

We consider both parametric and non-parametric formulations of time effect with the aim of identifying an appropriate modelling approach. Two smoothness priors are imposed on the temporal effects including first-order and second-order random walks (Knorr-Held 2000; Rue \& Held 2005). Bayesian computation was carried out via integrated nested Laplace approximation (INLA) (Rue, Martino \& Chopin 2009) which is computationally more efficient than the well-known Markov chain Monte Carlo approach. Applications of space-time modelling using INLA can be found in Schrödle \& Held (2011a,b) and Schrödle, Held, Riebler et al. (2011).

\subsection{Overall model}

Consider a study region discretized into $I$ regular or irregular regions (grid cells or SLAs). Let $y_{i j t}$ denote the event outcome of the $j$-th individual in the $i$-th region at the $\boldsymbol{t}$-th time interval, with individual characteristics of interest $\boldsymbol{x}_{\boldsymbol{i j}}=\left(x_{i j}^{(1)}, \ldots, x_{i j}^{(r)}\right)$, where $i=1, \ldots, I, j=1, \ldots, N$ and $t=1, \ldots, T$. Here $y_{i j t}$ is a binary response that follows a Bernoulli distribution with probability of disease $p_{i j t}$, which can be written as

$$
y_{i j t} \mid\left\{\boldsymbol{x}_{\boldsymbol{i j}}, \boldsymbol{\xi}_{\boldsymbol{i}}, \boldsymbol{\nu}_{\boldsymbol{t}}\right\} \stackrel{\text { ind }}{\sim} \operatorname{Bern}\left(p_{i j t}\right),
$$


where $\boldsymbol{\xi}_{\boldsymbol{i}}=\left\{u_{i}, v_{i}\right\}$ denote the random effects modelled on the regions and $\boldsymbol{\nu}_{\boldsymbol{t}}$ denotes the random effects modelled on the temporal aggregation.

In the context of the motivating dataset described above, $y_{i j t}$ denotes the Bernoulli outcome of advanced breast cancer for the $j$-th individual in the $i$-th region at the $t$-th time interval and $p_{i j t}$ denotes the individual risk of advanced breast cancer for the $j$-th individual in the $i$-th region at the $t$-th time interval. In the spirit of Jackson, Best \& Richardson (2008), the individual risk is modelled via a logistic regression model with inclusion of patient characteristics of interest and spatial and temporal random effects. We consider several models in which different assumptions for the time trend are specified, which are presented briefly in Table 1.

[Table 1 about here.]

\subsubsection{Linear time trend}

The first model follows the classical parametric formulation proposed by Bernardinelli, Clayton, Pascutto et al. (1995) which assumes a linear time trend for each region. The model (M1) is as follows,

$$
\operatorname{logit}\left(p_{i j t}\right)=\mu+\sum_{r} \alpha_{r} x_{i j}^{(r)}+u_{i}+v_{i}+\left(\beta+\delta_{i}\right) t
$$

where $\mu$ denotes the intercept, $\alpha_{r}$ denotes the covariate effect of the $r$-th covariate $x_{i j}, u_{i}$ and $v_{i}$ denote unstructured and spatially-structured random effects respectively (Besag, York \& Mollié 1991), that are both modelled on the regions, $\beta$ denotes a main linear time trend that represents the global time effect, $\delta_{i}$ is a differential trend for each region $i$ that identifies the interaction between time and space, and $t$ is the time interval.

Here, $\boldsymbol{u}=\left(u_{1}, u_{2}, \ldots, u_{I}\right)$ is a set of area-specific random effects that are assumed to be independent and identically distributed (i.i.d.). We model $u_{i} \sim$ $\operatorname{Normal}\left(0,1 / \tau_{u}\right)$, representing exchangeable random baseline risks for each region. The precision parameter $\tau_{u}$ is assigned a Gamma distribution $\tau_{u} \sim \operatorname{Gamma}\left(a_{u}, b_{u}\right)$ (Bernardinelli, Clayton, Pascutto et al. 1995).

The component $\boldsymbol{v}=\left(v_{1}, v_{2}, \ldots, v_{I}\right)$ is a set of area-specific spatially structured random effects such that spatial structure is induced via conditional autoregression (Besag, York \& Mollié 1991; Besag \& Kooperberg 1995). The full conditional for $v_{i}$ depends solely on the random effects of neighbouring areas. The intrinsic conditional autoregression 
(ICAR) specification (Besag, York \& Mollié 1991) is given by

$$
v_{i} \mid \boldsymbol{v}_{-i}, \tau_{v} \sim \mathcal{N}\left(\frac{1}{m_{i}} \sum_{i \sim k} v_{k}, \frac{1}{m_{i} \tau_{v}}\right),
$$

where $m_{i}$ is the number of neighbours of region $i, \boldsymbol{v}_{-i}$ denotes all elements in $\boldsymbol{v}$ except for $v_{i}$, and $i \sim k$ indicates that the two regions are neighbours that share a common boundary. The unknown precision parameter $\tau_{v}$ determines the strength of dependence between the parameters $v_{i}$ and $v_{k}$, and is assigned a Gamma distribution $\tau_{v} \sim \operatorname{Gamma}\left(a_{v}, b_{v}\right)$ (Bernardinelli, Clayton, Pascutto et al. 1995).

The random effects $\delta$ capture the interaction between the overall linear time trend $\beta$ and the regional random effects $\boldsymbol{u}$ and $\boldsymbol{v}$. The effect $\delta_{i}$ can also be defined as the difference between the region-specific time trend and the global time trend $\beta$. A negative $\delta_{i}$ indicates the slope of region $i$ is less steep than the main time trend $\beta$, whilst a positive $\delta_{i}$ implies that the slope of region $i$ is steeper than the main time trend. Since the spatial structure has been modelled using $v$, we assume that $\delta_{i}$ is spatially unstructured where $\delta_{i} \sim \operatorname{Normal}\left(0,1 / \tau_{\delta}\right)$ and $\tau_{\delta}$ is assigned a Gamma distribution $\tau_{\delta} \sim \operatorname{Gamma}\left(a_{\delta}, b_{\delta}\right)$. However, we note that prior specification of $\delta$ may follow a conditional autoregressive structure (Bernardinelli, Clayton, Pascutto et al. 1995; Schrödle \& Held 2011a). To avoid high correlation between $u_{i}, v_{i}$ and $\delta_{i}$, the time variable $t$ is centred at zero, as proposed by Bernardinelli, Clayton, Pascutto et al. (1995). The parameters estimated by INLA are $\boldsymbol{\theta}=\{\mu, \boldsymbol{\alpha}, \boldsymbol{u}, \boldsymbol{v}, \beta, \boldsymbol{\delta}\}$ and the hyperparameters are represented by $\boldsymbol{\psi}=\left\{\tau_{\alpha}, \tau_{u}, \tau_{v}, \tau_{\delta}\right\}$.

\subsubsection{Non-parametric time trend}

The following models adopt a dynamic non-parametric formulation which relaxes the linearity assumption in model M1 (Knorr-Held 2000). The linear predictor is

$$
\operatorname{logit}\left(p_{i j t}\right)=\mu+\sum_{r} \alpha_{r} x_{i j}^{(r)}+u_{i}+v_{i}+\phi_{t}+\gamma_{t}
$$

Here $\mu, \boldsymbol{\alpha}, \boldsymbol{u}$ and $\boldsymbol{v}$ follow the same definition in model M1 (1), and $\phi_{t}$ and $\gamma_{t}$ denote unstructured and temporally-structured random effects respectively that are modelled on the time periods. The main effects $v$ and $\gamma$ are centred around zero to ensure identifiability of the intercept $\mu$.

The component $\phi=\left(\phi_{1}, \phi_{2}, \ldots, \phi_{t}\right)$ is a set of time-specific random effects that are assumed to be independent and identically distributed. We model $\phi_{t} \sim \operatorname{Normal}\left(0,1 / \tau_{\phi}\right)$, representing exchangeable effect for each time interval. The precision parameter $\tau_{\phi}$ is assigned a Gamma distribution $\tau_{\phi} \sim \operatorname{Gamma}\left(a_{\phi}, b_{\phi}\right)$. 
The temporally-structured component $\gamma=\left(\gamma_{1}, \gamma_{2}, \ldots, \gamma_{t}\right)$ is assumed to follow a random walk of first-order (RW1) or a random walk of second-order (RW2) (Besag \& Kooperberg 1995; Rue \& Held 2005). The prior density of RW1 can be written as

$$
\pi\left(\gamma \mid \tau_{\gamma}\right) \propto \exp \left(-\frac{\tau_{\gamma}}{2} \sum_{t=2}^{T}\left(\gamma_{t}-\gamma_{t-1}\right)^{2}\right) .
$$

We name this model as M2. The density of RW2 is

$$
\pi\left(\gamma \mid \tau_{\gamma}\right) \propto \exp \left(-\frac{\tau_{\gamma}}{2} \sum_{t=3}^{T}\left(\gamma_{t}-2 \gamma_{t-1}+\gamma_{t-2}\right)^{2}\right)
$$

and this model is named as M3. We assign $\tau_{\gamma} \sim \operatorname{Gamma}\left(a_{\gamma}, b_{\gamma}\right)$. The parameters of interest are thus $\boldsymbol{\theta}=\{\mu, \boldsymbol{\alpha}, \boldsymbol{u}, \boldsymbol{v}, \boldsymbol{\phi}, \boldsymbol{\gamma}\}$ with hyperparameters represented by $\boldsymbol{\psi}=$ $\left\{\tau_{\alpha}, \tau_{u}, \tau_{v}, \tau_{\phi}, \tau_{\gamma}\right\}$.

\subsubsection{Non-parametric time trend with space-time interaction effect}

An interaction between space and time is obtained by expanding models M2 and M3 using the following specification

$$
\operatorname{logit}\left(p_{i j t}\right)=\mu+\sum_{r} \alpha_{r} x_{i j}^{(r)}+u_{i}+v_{i}+\phi_{t}+\gamma_{t}+\epsilon_{i t}
$$

The inclusion of the interaction effect in models M2 and M3 results in models M4 and M5, respectively. In this study, we considered the interaction of unstructured spatial and unstructured temporal effects. It follows that $\epsilon_{i t} \sim \operatorname{Normal}\left(0,1 / \tau_{\epsilon}\right)$. The precision parameter $\tau_{\epsilon}$ is assigned a Gamma distribution $\tau_{\epsilon} \sim \operatorname{Gamma}\left(a_{\epsilon}, b_{\epsilon}\right)$. We note that other specifications for the interaction term are possible (Knorr-Held 2000) but here we assume that the two unstructured effects $u_{i}$ and $\phi_{t}$ interact. This assumption is also motivated by the fact that the inclusion of an interaction effect did not appear to improve model goodness-of-fit, as discussed in Section 4.1.

Similarly to model M2, the main effects $\boldsymbol{v}$ and $\gamma$ are centred around zero to ensure identifiability of the intercept $\mu$. Note that no constraint is required for the identifiability of the interaction term $\epsilon$ as the structural matrix of the Type I interaction does not induce a rank deficiency (Schrödle \& Held 2011b).

The parameters of interest are now $\boldsymbol{\theta}=\{\mu, \beta, \boldsymbol{\alpha}, \boldsymbol{u}, \boldsymbol{v}, \boldsymbol{\phi}, \boldsymbol{\gamma}, \boldsymbol{\epsilon}\}$ and the vector of hyperparameters is $\boldsymbol{\psi}=\left\{\tau_{\alpha}, \tau_{u}, \tau_{v}, \tau_{\phi}, \tau_{\gamma}, \tau_{\epsilon}\right\}$. 


\subsection{Computation}

We adopt the integrated nested Laplace approximation (INLA) approach proposed by Rue, Martino \& Chopin (2009), which performs approximate Bayesian inference for latent Gaussian models. The INLA approach is able to return accurate parameter estimates in relatively short computational time. The latent Gaussian models can be defined as a Bayesian hierarchical model with three levels. The first level is the observational equation $\boldsymbol{y} \mid \boldsymbol{\theta}, \boldsymbol{\psi} \sim \pi(\boldsymbol{y} \mid \boldsymbol{\theta}, \boldsymbol{\psi})$, where $\boldsymbol{y}$ denotes the observations that depend on the latent field $\boldsymbol{\theta}$ and hyperparameters $\boldsymbol{\psi}$. The second level is $\boldsymbol{\theta} \mid \boldsymbol{\psi} \sim N\left(\boldsymbol{\mu}(\boldsymbol{\psi}), \boldsymbol{Q}(\boldsymbol{\psi})^{-1}\right)$, where $\boldsymbol{\theta}$ contains all components of the latent Gaussian field. At the third level, $\boldsymbol{\psi} \sim \pi(\boldsymbol{\psi})$ denotes the prior distribution for the parameters. $\boldsymbol{Q}(\psi)$ is the precision matrix of the Gaussian random vector $\boldsymbol{\theta}$, which is sparse. The posterior density can be written as

$$
\pi(\boldsymbol{\theta}, \boldsymbol{\psi} \mid \boldsymbol{y}) \propto \pi(\boldsymbol{\psi}) \pi(\boldsymbol{\theta} \mid \boldsymbol{\psi}) \prod_{i \in I} \pi\left(y_{i} \mid \theta_{i}, \boldsymbol{\psi}\right)
$$

The components of the latent field, $\boldsymbol{\theta}$, are assigned Gaussian priors with precision matrix $Q(\psi)$, resulting in a latent Gaussian field. The latent field is controlled by a few hyperparameters $\psi$ which vary according to different models, as previously described.

The main goal is to estimate the desired posterior marginals

$$
\pi\left(\theta_{i} \mid \boldsymbol{y}\right)=\int \pi\left(\theta_{i} \mid \boldsymbol{\psi}, \boldsymbol{y}\right) \pi(\boldsymbol{\psi} \mid \boldsymbol{y}) d \boldsymbol{\psi}
$$

while the posterior marginals of $\boldsymbol{\psi}$ are approximated by

$$
\pi\left(\psi_{i} \mid \boldsymbol{y}\right)=\int \pi(\boldsymbol{\psi} \mid \boldsymbol{y}) d \boldsymbol{\psi}_{-i}
$$

where $\boldsymbol{\psi}_{-i}$ denotes all elements in $\boldsymbol{\psi}$ except for $\psi_{i}$. Nested approximations and numerical integration are used to integrate out $\psi$ in order to estimate (2) and (3). The Laplace approximation (Tierney \& Kadane 1986) to the posterior of hyperparameters can be written as

$$
\left.\tilde{\pi}(\boldsymbol{\psi} \mid \boldsymbol{y}) \propto \frac{\pi(\boldsymbol{\theta}, \boldsymbol{\psi}, \boldsymbol{y})}{\tilde{\pi}_{G}(\boldsymbol{\theta} \mid \boldsymbol{\psi}, \boldsymbol{y})}\right|_{\boldsymbol{\theta}=\boldsymbol{\theta}^{*}(\boldsymbol{\psi})},
$$

where $\tilde{\pi}_{G}(\boldsymbol{\theta} \mid \boldsymbol{\psi}, \boldsymbol{y})$ is the Gaussian approximation to the full conditional of $\boldsymbol{\theta}$ and $\boldsymbol{\theta}^{*}(\boldsymbol{\psi})$ is the mode of the Gaussian approximation for each $\boldsymbol{\theta}$. Posterior marginals for the latent variables $\boldsymbol{\theta}$ and the hyperparameters $\boldsymbol{\psi}$ are both computed via numerical integration. The posterior marginals can be used to compute summary statistics of interest, such as posterior means, variances or quantiles. 


\subsection{Priors}

To complete the Bayesian specification, hyperprior distributions must be assigned to all precision components described above. Since the mean of the fixed effects $\mu, \beta$ and $\boldsymbol{\alpha}$ are unknown, these parameters are assigned a Normal distribution with large variance, $\operatorname{Normal}\left(0,0.001^{-1}\right)$. The precision parameter of the i.i.d. random effects, including $\tau_{u}, \tau_{\delta}$, $\tau_{\phi}$ and $\tau_{\epsilon}$, are assigned Gamma(1,0.01) throughout the study for ease of comparison. In this work, we have chosen Gamma(1,0.01) as motivated by its use in Schrödle \& Held (2011b) and Schrödle, Held, Riebler et al. (2011). However, we note that this prior choice might not be ideal for our case study and could be improved by attempting a range of priors. A discussion of possible confounding between the unstructured and structured spatial effects caused by prior choices can be found in Bernardinelli, Clayton, Pascutto et al. (1995) and Simpson, Martins, Riebler et al. (2014).

The priors imposed on the spatial component $(v)$ and the temporal component $(\gamma)$, discussed in Section 3.1, are classified as intrinsic Gaussian Markov random field (IGMRF) priors. It is known that the hyperprior chosen for the precision parameters has an impact on the degree of smoothness of the resulting spatial or temporal field as well as the posterior distribution. The problem here is that a specific fixed hyperprior for the precision parameter does not give the same interpretations for different IGMRF priors. To ensure fair comparison between different models, we adopt the technique of scaling IGMRF priors via R-INLA recently proposed by Sørbye \& Rue (2014). By scaling the priors, a fixed hyperprior for the precision parameter results in a similar interpretation for different types of IGMRF priors. The approach is implemented by specifying 'scale.model=TRUE' in R-INLA (Sørbye 2013) which scales the priors to have a generalized variance equal to 1. As a result, the precision parameters of different IGMRF priors have similar interpretation. It is now reasonable to assign the same fixed hyperprior (chosen to be $\operatorname{Gamma}(1,0.01)$ ) to precision parameters of different IGMRFs in a regression model. Using the notion of scale, Simpson, Martins, Riebler et al. (2014) introduce a new concept for constructing prior distributions which may be of interest to R-INLA users. As an illustration, see Appendix A for the R-INLA code used to fit models M1 to M5 at the 3-year aggregation level and the SLA level.

\subsection{Model selection criteria}

In the following section, we discuss two approaches for model selection, i.e. the DIC and logarithmic score (LS). The model performance in terms of estimation of the fixed and random effects is also discussed. The DIC (Spiegelhalter, Best, Carlin et al. 2002) is a tool of Bayesian model choice for selecting the most parsimonious model after penalizing for model 
complexity. It is defined as two times the mean of the deviance minus the deviance of the mean. For a likelihood $\pi(\boldsymbol{y} \mid \boldsymbol{\theta}, \boldsymbol{\psi})$, the deviance is $\mathrm{D}(\boldsymbol{\theta}, \boldsymbol{\psi})=-2 \log \pi(\boldsymbol{y} \mid \boldsymbol{\theta}, \boldsymbol{\psi})$. A smaller DIC indicates a better trade-off between model fit and complexity. The DIC works well as a method for screening alternative formulations in order to provide an indication of the relative fit of a set of candidate models. Candidate models receiving DIC values within $1-2$ of the 'best' model deserve consideration, while $3-7$ have considerably less support. The DIC is one of the most popular methods for comparing hierarchical models. Its use for comparison of spatio-temporal Bayesian hierarchical models is also common (Norton 2008). However, we note that the DIC has been criticized (Celeux, Forbes, Robert et al. 2006; Aitkin 2010) and can be problematic in models with many random effects (Plummer 2008), and thus should be used with care.

The logarithmic score (LS) for each model is computed to assess the predictive quality of these models (Rue, Martino \& Chopin 2009; Gneiting \& Raftery 2007). Each model is assigned a numerical score based on the predictive distribution using the cross-validated scoring rules. For discrete observations $Y_{i j t}$, the LS is defined as

$$
\mathrm{LS}=-\log \left(\pi_{y_{i j t}}\right)
$$

where $\pi_{y_{i j t}}=\operatorname{Prob}\left(Y_{i j t}=y_{i j t} \mid \boldsymbol{y}_{-i j t}\right)$ denotes the cross-validated predictive probability mass at the observed event. A smaller LS indicates a better predictive quality of the model. See Gneiting \& Raftery (2007) for detailed calculation of the score.

\section{Results}

We started the analyses by fitting all model combinations from the six covariates (patients' characteristics). This stage involved fitting a logistic regression model to the data in order to identify covariates that contributed significantly to the individual risk. The spatial and temporal effects were not included in the models in these preliminary analyses as the aim was to investigate the impact of each covariate on the individual risk of advanced breast cancer. A total of 63 models were fitted to the breast cancer data, containing all the possible combinations of the six variables. That is, all the combinations of one variable (6 models), two variables (15 models), three variables (20 models), four variables (15 models), five variables (6 models) and six variables (1 model). The combination of the covariates are illustrated in Table 2.

We examined the DIC and the regression parameters of each model. The DIC values were in the range $[11358.98,11392.22]$. The model fitting procedures showed that the inclusion of variables for which the $90 \%$ credible intervals of the corresponding coefficients 
do not contain zero had decreased the DIC substantially. In view of this, it was sensible to only keep significant variables in the model. The model with covariate IRSD had the smallest DIC value and parameter estimates that do not contain zero in the $90 \%$ credible interval. The difference between the smallest DIC value and the second smallest DIC value is 3.7, which suggests a significant improvement in the model with IRSD. We thus proceeded with further analyses using this variable.

[Table 2 about here.]

To evaluate the impact of modelling the temporal effects at various levels of aggregation, six time periods were considered, comprising 3-year, 2.5-year, 2-year, 1.5-year, 1-year, and 0.5 -year periods. Two levels of spatial aggregation were chosen to model the random effects over the space, namely the grid level (900 grid cells) and the SLA level (308 SLAs). In this section, we present the results of fitting all five models at various levels of spatial and temporal aggregation.

\subsection{Goodness-of-fit and model selection}

The DIC and LS for models M1 to M5 at all six levels of temporal aggregation and two levels of spatial aggregation are presented in Figure 2. A smaller score indicates a more favourable model. We note that both scores show very similar results in terms of model performance. It can be seen that spatial aggregation at the SLA level resulted in smaller DIC and LS values than those at the grid level. Note that the scores for each model remained relatively consistent across the varying level of temporal aggregation. This suggests that the impact of temporal aggregation was indistinguishable based on the DIC and LS values presented here. Compared to the best DIC value (11358.98), presented earlier, after taking into account the spatial and temporal random effects, model M1 at the 3-year level showed a slight improvement in goodness-of-fit, resulting in a DIC score of 11357.87. Nevertheless, the differences in the DIC and LS scores for different models are very small. For instance, at the SLA level, the DIC score centres around 11360 for all models and does not differ by more than 5 between models. Similarly, the differences in the LS between models are very small in which they are only visible at the fourth decimal place.

[Figure 2 about here.]

Comparison of the two levels of spatial aggregation showed that the models with spatial effects modelled at the SLA level consistently performed better than the models fitted at the grid level. Note that the covariate IRSD included in the model was measured at the SLA level, rather than the grid level. The residual risk at the SLA level could possibly be better identified compared to the analogous risk at the grid level due to the nature of the covariate itself. 
Comparison across the five models indicated that model M1 was favoured among all the considered levels of spatial and temporal aggregation. We note that model M1 is a parametric model which contains a main linear time trend and a potentially different trend for each region. A possible explanation for this result is that the parametric time trend in the dataset remains rather similar across the varying temporal aggregation. Here models M2 to M5 are non-parametric models that assume a random walk pattern for the temporal effects. The findings suggest that a non-parametric formulation for the time trend in the case study data may not be appropriate as it does not improve the goodness-of-fit of the model. Further inspection of the results are made below to facilitate inference about the impact of different model formulations on model estimation.

\subsection{Estimation of fixed effects}

The standard deviation of the posterior estimates of regression parameters can provide guidance on how the estimation of the fixed effects changes when different models are considered across various levels of temporal aggregation. A larger standard deviation indicates a larger spread of the posterior distribution. The covariate IRSD included in the models has five categories and thus four dummy variables were created, namely IRSD1, IRSD2, IRSD3 and IRSD4. Figure 3 presents the standard deviation of the posterior estimates of IRSD1 at all six levels of temporal aggregation and two levels of spatial aggregation. The results for IRSD2, IRSD3 and IRSD4 are omitted here as they showed similar patterns to those produced by IRSD1. The results suggest that models M2 and M3, at the grid level, had higher precision in the estimation of regression parameters at all levels of temporal aggregation and remained consistent regardless of the changes in the aggregation levels.

The models at the SLA level, however, are slightly more sensitive to the varying level of temporal aggregation in estimating the regression parameters. It can be seen that there are some differences in the standard deviation of the estimated regression parameters across the different aggregation levels, with model M4 at the 2.5-year level producing the highest precision of estimation. In general, the precision of estimation at the SLA level was consistently higher than that at the grid level. We recall that IRSD is an area-level covariate measured at the SLA level for the individuals within each SLA. Parameter estimation of the models at the SLA level was more precise, possibly due to the closer compatibility between the scale at which the variable was collected and the scale at which the inference was made. However, we note that the differences in the standard deviation between models are very minor, in which they are only visible at the third and fourth decimal places, for the SLA and grid levels, respectively. Therefore, comparison of the models using the standard deviation of the fixed effects may not be relevant in practice for our case study. 
[Figure 3 about here.]

The posterior probabilities $\left(\mathrm{P}\left(\alpha_{p}<0\right)\right)$ for the regression parameters IRSD1, IRSD2, IRSD3, IRSD4 and TIME are presented in Table 3. TIME is only applicable for model M1 whereas IRSD1 to IRSD4 are applicable to all five models. The posterior probabilities provide evidence for the coefficients of the regression parameters to be less than zero given the data. For instance, there is some evidence that the coefficients of IRSD3 and IRSD4 are less than zero given that the corresponding posterior probabilities are over 0.8 for all models.

Looking at the posterior probabilities at the grid and SLA levels individually, the 'Max-Min' column shows the difference in the maximum and the minimum values across different levels of temporal aggregation. At the grid level, temporal aggregation had almost no influence on the posterior probabilities as only negligible differences were observed. At the SLA level, temporal aggregation had a slightly greater impact on the posterior probabilities, but the effect was still arguably small. For IRSD1, IRSD3 and TIME, the parameters estimated at the grid level had slightly larger posterior probabilities than those estimated at the SLA levels. The posterior probabilities of IRSD2 and IRSD4, however, were somewhat larger at the SLA level. These results clearly indicate that spatial aggregation had an impact on parameter estimation.

[Table 3 about here.]

\subsection{Estimation of random effects}

Box plots of the standard deviation of the random effects for models M1 to M5 at the grid level are presented in Figures 4, 5 and 6. Here we omit the results produced at the SLA level. We note that each model has different specifications of random effects. The plots provide an insight into the precision of estimation of the random effects for different model formulations at various levels of temporal aggregation. These results indicate that temporal aggregation had little impact on the precision of estimation of the random effects, since the standard deviations of different random effects remained fairly consistent across the various levels of aggregation for each of the models. This is due to the fact that the IGMRF priors employed in the models were scaled to have a similar interpretation for the precision parameter, as described in Section 3.3.

Recall the formulations of models M2 and M3, which differ with respect to the prior for temporal smoothing in that model M2 employs a first-order random walk whereas model M3 imposes a second-order random walk. The box plots of standard deviation of the temporally structured random effects $(\gamma)$ in model M3 have a larger length compared to those of model M2, suggesting a larger variation and a smaller precision. This larger variation of $\gamma$ in model 
M3 is possibly induced by the inclusion of second-order neighbours in model M3. Similarly for models M4 and M5 which have different temporal smoothness priors, the box plots of standard deviation of $\gamma$ in model M5 have a larger length compared to those of model M4.

[Figure 4 about here.]

[Figure 5 about here.]

[Figure 6 about here.]

\section{Discussion}

In this study, we investigate several model formulations for modelling individual disease outcomes via Bayesian hierarchical spatio-temporal modelling. We examine both parametric (model M1) and non-parametric (models M2 to M5) formulations for the temporal effect at various levels of spatial and temporal aggregation. These models take into account individual characteristics, aggregate information, spatial random effects, and temporal random effects. Two levels of aggregation used to model the spatial effect are the grid and SLA levels. The grid partitions used are regular lattices that are finer than the SLAs, in which the discretization of the study region results in 900 artificial grid cells as opposed to 308 SLAs that are census regions. We propose the grid partitions as it is more natural and reasonable to implement the ICAR specification for geographical areas that are of similar size and arranged in a regular pattern (Wakefield 2007). Motivated by Leyk, McCormick \& Nuckols (2011) who argue that varying temporal aggregation appears to affect variable selection and coefficient values, we are interested in studying the impact of model formulations on model performance and precision of estimation, while considering the possible impact of spatial and temporal aggregation. Six levels of temporal aggregation were chosen to model the temporal effect for the breast cancer dataset, namely 3-year, 2.5-year, 2-year, 1.5-year, 1-year, and 0.5-year periods. Due to the lack of time-related variables, the current study was not able to identify the relation between the temporal aggregation and variable selection.

With regard to the choice of model formulation, model M1, a parametric model with a main linear time trend and a potentially varying trend for each region, resulted in the best fit among all considered models. The DIC and LS values suggested that the impact of temporal aggregation was indistinguishable using the aggregation levels considered in this study. In contrast, spatial aggregation had an impact on model goodness-of-fit, as supported by the findings in Kang, McGree, Baade et al. (2014).

In terms of the estimation of regression parameters, models M2 and M3 appeared to be favoured at the grid level, at which both the first-order and second-order random walk priors 
for temporal smoothing were shown to produce a slightly better precision of estimation as compared to other models. We note that these differences were very small and may not be relevant in practice. In general, the models at the SLA level were slightly sensitive towards the changes in the levels of temporal aggregation. However, temporal aggregation had little impact on the precision of estimation at the grid level.

The findings from Section 4.3 have provided an understanding about the precision of estimation of various random effects for different models. Given the unvarying standard deviation across different levels of temporal aggregation, it was reasonable to compare the models across different aggregation levels. However, the results did not allow for the selection of the most appropriate model for the case study data.

As discussed, the goodness-of-fit and precision of parameter estimation for the SLAbased models were slightly better than those of the grid-based models. We acknowledge that the covariate IRSD is a SLA-measured variable, and thus the spatial effect is possibly better identified at the same scale as the data collected, which may explain the better performance of the models at the SLA level. Similarly to SLAs that are purely administrative regions, the grid partitions are artificial boundaries and so do not reflect the access to health services or sense of community (Kang, McGree, Baade et al. 2014). However, the researcher has the power to manipulate the spatial resolution so as to obtain an appropriate grid cell size to suit an inferential aim.

Focussing primarily on the impact of different levels of temporal aggregation, this study has not taken the possible age-group effects into consideration due to data sparseness and insufficient data to include a cohort effect. We note that Bayesian age-period-cohort model (Knorr-Held \& Rainer 2001; Riebler \& Held 2010; Held \& Riebler 2012) may serve as a good approach to smooth the effects of age, period and cohort in order to improve estimation and facilitate prediction. This will be an avenue for future investigation for other datasets.

In summary, the findings of the case study have revealed that different model formulations produce different model performance. Specifically, a model with a classical parametric formulation that assumes a linear time trend gave the best fit for our dataset. This in turn suggests the importance of employing the most suitable model for spatio-temporal analyses by exploring a range of model formulations. This is particularly important from a policy maker's perspective where the interpretation of the model results may be crucial to effective policy decisions with regard to a disease outcome.

\section{Appendix A: R-INLA code}

As an illustration, here we present the R-INLA code used for fitting models M1 to M5 at the 3-year aggregation level and the SLA level. 


\section{library ( INLA)}

\#\# Scale the IGMRF-models \#\#

inla. set Option ( s cale model. default=TRUE)

\#\# 3-year temporal aggregation level \#\#

time.struct $<-$ time_36month

time. iid $<-$ time_36month

time_main_centered $<-$ time_36month_centered

interact $<-$ region. iid $*$ time. iid

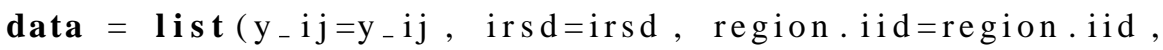

region. struct=region. struct, time. struct=time. struct, time . iid=time . iid, time _main_centered=time _main _ centered, interact $=$ interact)

\#\# Model M1 \#\#

formula $=y_{-} i j \sim 1+i r s d+f(r e g i o n . s t r u c t, \operatorname{model}="$ besag", graph="sla.graph", adjust . for . con . comp=FALSE, hyper=list ( theta $=\mathbf{l i s t}($ prior $=" \operatorname{loggamma} ", \operatorname{param}=\mathbf{c}(1,0.01))))+\mathrm{f}(\operatorname{region}$. iid , model=" iid", hyper $=\mathbf{l i} \mathbf{i} \mathbf{t}($ theta $=\mathbf{l}$ ist $($ prior $=" \operatorname{loggamma",}$ $\operatorname{param}=\mathbf{c}(1,0.01))))+$ time _main_centered + f(interact , model=" i id", hyper $=\mathbf{l}$ ist $($ thet $\mathbf{a}=\mathbf{l}$ ist $($ prior $=" \operatorname{loggamma",param=}$ c $(1,0.01))))$

result $1=$ inla (formula, family="binomial", data=data, Ntrials $=1$, control. in 1 a $=$ list ( strategy="gaussian"), control. compute $=\mathbf{l}$ ist $($ dic=TRUE, cpo=TRUE), verbose=FALSE, control . fixed $=\mathbf{l}$ is t $($ mean $=0, \operatorname{prec}=0.001))$

summary ( result 1 )

\#\# Model M2 \#\# 


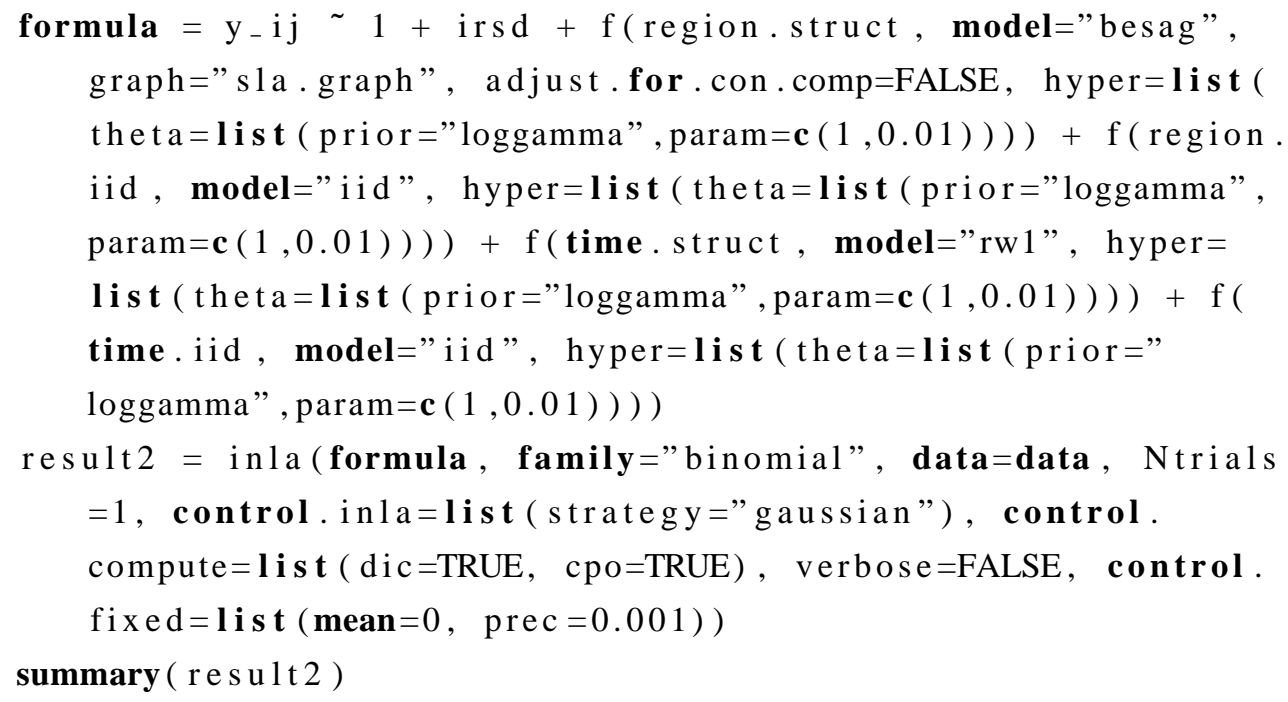

\#\# Model M3 \#\#

formula $=y_{-} i j \sim 1+i r s d+f(r e g i o n$. struct, model="besag", graph="sla.graph", adjust . for . con . comp=FALSE, hyper=list ( thet a $=\mathbf{l i s t}(\operatorname{prior}=" \operatorname{loggamma} ", \operatorname{param}=\mathbf{c}(1,0.01))))+\mathrm{f}(\operatorname{region}$. iid , model=" iid" , hyper $=\mathbf{l i s t}($ theta=list $($ prior $=" \log$ gamma", $\operatorname{param}=\mathbf{c}(1,0.01))))+\mathrm{f}($ time $\cdot \operatorname{struct}, \mathbf{m o d e l}=" \mathrm{rw} 2 "$, hyper $=\mathbf{l} \mathbf{i s t}$ $($ thet a $=\mathbf{l i s t}($ prior $=" \log g a m m a ", \operatorname{param}=\mathbf{c}(1,0.01))))+\mathrm{f}($ time. iid , model=" i id" , hyper $=\mathbf{l i s} \mathbf{t}($ theta $=\mathbf{l}$ ist $($ prior $=" \operatorname{loggamma",}$ $\operatorname{param}=\mathbf{c}(1,0.01))))$

result 3 = inla (formula, family="binomial", data=data, Ntrials $=1$, control. inla =list ( strategy="gausian”), control. compute $=\mathbf{l}$ ist $(\mathrm{dic}=$ TRUE, cpo=TRUE), verbose=FALSE, control . fixed $=\mathbf{l}$ is t $($ mean $=0, \operatorname{prec}=0.001))$

summary ( result 3 ) 
formula $=y_{-} \mathrm{ij} \sim 1+\mathrm{irsd}+\mathrm{f}($ region. struct, model="besag", graph="sla.graph", adjust.for. con . comp=FALSE, hyper=list ( thet a $=\mathbf{l i s t}(\operatorname{prior}=" \log$ amma" $\operatorname{param}=\mathbf{c}(1,0.01))))+\mathrm{f}(\operatorname{region}$. iid , model=" iid", hyper $=\mathbf{l i s} \mathbf{t}($ theta $=\mathbf{l}$ ist $($ prior $=" \operatorname{loggamma",}$ $\operatorname{param}=\mathbf{c}(1,0.01))))+\mathrm{f}($ time $\cdot$ struct , model="rw1", hyper $=\mathbf{l i s t}$ $($ theta $=\mathbf{l i s} \mathbf{t}($ prior $=" \operatorname{loggamma} ", \operatorname{param}=\mathbf{c}(1,0.01)))+\mathrm{f}($ time . iid , model=" i id", hyper $=\mathbf{l}$ ist $($ theta $=\mathbf{l}$ ist $($ prior $=" \operatorname{loggamma",}$ $\operatorname{param}=\mathbf{c}(1,0.01))))+\mathrm{f}($ interact , model=" iid", hyper=list ( theta $=\mathbf{l i s} \mathbf{t}(\operatorname{prior}=" \log g a m m a ", \operatorname{param}=\mathbf{c}(1,0.01))))$ result4 = inla (formula, family="binomial", data=data, Ntrials $=1$, control. inla =list ( strategy="gaussian"), control. compute $=\mathbf{l}$ ist $(\mathrm{dic}=$ TRUE, cpo=TRUE), verbose=FALSE, control . fixed $=\mathbf{l i s t}(\operatorname{mean}=0, \operatorname{prec}=0.001))$

summary ( result 4 )

\section{\#\# Model M5 \#\#}

formula $=y_{-} i j \sim 1+i r s d+f($ region. struct, model="besag", graph="sla.graph", adjust . for . con . comp=FALSE, hyper =list ( thet a $=\mathbf{l i s t}(\operatorname{prior}=" \operatorname{loggamma} ", \operatorname{param}=\mathbf{c}(1,0.01))))+\mathrm{f}(\operatorname{region}$. iid , model=" i id" , hyper $=\mathbf{l}$ ist $($ thet a $=\mathbf{l}$ ist $($ prior $=" \operatorname{loggamma",}$ param $=\mathbf{c}(1,0.01))))+\mathrm{f}($ time . struct , model=" rw2", hyper $=\mathbf{l i s} \mathbf{t}$ $($ theta $=\mathbf{l i s} \mathbf{t}(\operatorname{prior}=" \operatorname{loggamma} ", \operatorname{param}=\mathbf{c}(1,0.01))))+\mathrm{f}($ time. iid , model=" iid", hyper $=\mathbf{l i s} \mathbf{t}($ theta $=\mathbf{l}$ ist $($ prior $=" \operatorname{loggamma",}$ $\operatorname{param}=\mathbf{c}(1,0.01))))+\mathrm{f}($ interact, model="iid", hyper=list ( thet a $=\mathbf{l}$ ist $($ prior $=" \log g a m m a ", \operatorname{param}=\mathbf{c}(1,0.01))))$

result5 = inla (formula, family="binomial", data=data, Ntrials $=1$, control. inla $=$ list ( strategy="gausian"), control. compute $=\mathbf{l i s t}$ ( dic=TRUE, cpo=TRUE), verbose=FALSE, control . fixed $=\mathbf{l i s t}($ mean $=0, \operatorname{prec}=0.001))$ summary ( result 5 )

\section{References}

ABELLAN, J.J., RichARDSON, S. \& BEST, N. (2008). Use of space-time models to investigate the stability of patterns of disease. Environ Health Persp 116, 1111.

ADHIKARI, P. (2006). Socio-economic indexes for areas: Introduction, use and future directions. Canberra: Australian Bureau of Statistics. 
AIHW (2004). Rural, regional and remote health: A guide to remoteness classifications. Canberra: Australian Institute of Health and Welfare AIHW Cat. No. PHE 53.

AitKIn, M. (2010). Statistical Inference: An Integrated Bayesian/Likelihood Approach. Chapman \& Hall/CRC.

Anselin, L., Syabri, I. \& Kho, Y. (2006). GeoDa: An introduction to spatial data analysis. Geogr Anal 38, 5-22.

BaAde, P.D., Turrell, G. \& Aitken, J.F. (2011). Geographic remoteness, area-level socio-economic disadvantage and advanced breast cancer: A cross-sectional, multilevel study. J Epidemiol Commun $H$ 65, 1037-43.

Bernardinelli, L., Clayton, D., Pascutto, C., Montomoli, C., Ghislandi, M. \& Songini, M. (1995). Bayesian analysis of space-time variation in disease risk. Stat Med 14, 2433-2443.

Besag, J. \& KoOPERberg, C. (1995). On conditional and intrinsic autoregressions. Biometrika 82, 73346.

Besag, J., York, J. \& Mollié, A. (1991). Bayesian image restoration, with two applications in spatial statistics. Ann I Stat Math 43, 1-59.

Bivand, R., Anselin, L., Berke, O., Bernat, A., Carvalho, M., Chun, Y., Dormann, C.F., Dray, S., Halbersma, R., Lewin-Koh, N. et al. (2011). spdep: Spatial dependence: Weighting schemes, statistics and models. $R$ package version 0.5-56, URL http://cran.rproject.org/web/packages/spdep/index.html .

Celeux, G., Forbes, F., Robert, C.P. \& Titterington, D.M. (2006). Deviance information criteria for missing data models. Bayesian Analysis 1, 651-673.

GNeiting, T. \& RAFTERY, A.E. (2007). Strictly proper scoring rules, prediction, and estimation. J Am Stat Assoc 102, 359-78.

Held, L. \& RiebleR, A. (2012). A conditional approach for inference in multivariate age-period-cohort models. Stat Methods Med Res 21, 311-329.

JACKSON, C., BEST, N. \& RiChARDSON, S. (2008). Hierarchical related regression for combining aggregate and individual data in studies of socio-economic disease risk factors. J R Stat Soc Ser A Stat Soc 171, $159-78$.

Kang, S.Y., McGree, J., BAade, P. \& Mengersen, K. (2014). An investigation of the impact of various geographical scales for the specification of spatial dependence. Journal of Applied Statistics , 1-24.

KNORR-HELD, L. (2000). Bayesian modelling of inseparable space-time variation in disease risk. Stat Med 19, 2555-2567.

Knorr-Held, L. \& Besag, J. (1998). Modelling risk from a disease in time and space. Stat Med 17, 2045-2060.

KnORR-Held, L. \& RAINER, E. (2001). Projections of lung cancer mortality in West Germany: a case study in Bayesian prediction. Biostatistics 2, 109-129.

KnorR-Held, L., RASSER, G. \& BECKER, N. (2002). Disease mapping of stage-specific cancer incidence data. Biometrics 58, 492-501.

Lawson, A.B. (2013). Bayesian Disease Mapping: Hierarchical Modeling in Spatial Epidemiology, vol. 32. Chapman and Hall/CRC.

LEYK, S., McCormick, B.J.J. \& Nuckols, J.R. (2011). Effects of varying temporal scale on spatial models of mortality patterns attributed to pediatric diarrhea. Spat Spatiotemporal Epidemiol 2, 91-101.

MACNAB, Y.C. \& GUSTAFson, P. (2007). Regression B-spline smoothing in Bayesian disease mapping: with an application to patient safety surveillance. Stat Med 26, 4455-4474.

Nobre, A.A., Schmidt, A.M. \& Lopes, H.F. (2005). Spatio-temporal models for mapping the incidence of malaria in Pará. Environmetrics 16, 291-304.

Norton, J.D. (2008). Spatiotemporal Bayesian Hierarchical Models, with Application to Birth Outcomes. ProQuest LLC. 
OCAÑA-Riola, R. (2007). The misuse of count data aggregated over time for disease mapping. Stat Med 26, 4489-4504.

OCAÑA-Riola, R. (2010). Common errors in disease mapping. Geospat Health 4, 139-154.

Plummer, M. (2008). Penalized loss functions for Bayesian model comparison. Biostatistics 9, 523-539.

Richardson, S., Abellan, J.J. \& Best, N. (2006). Bayesian spatio-temporal analysis of joint patterns of male and female lung cancer risks in Yorkshire (UK). Stat Methods Med Res 15, 385-407.

RICHARDSON, S. \& BEST, N. (2003). Bayesian hierarchical models in ecological studies of healthenvironment effects. Environmetrics 14, 129-47.

Riebler, A. \& Held, L. (2010). The analysis of heterogeneous time trends in multivariate age-periodcohort models. Biostatistics 11, 57-69.

Rue, H. \& Held, L. (2005). Gaussian Markov Random Fields: Theory and Applications, Monographs on Statistics and Applied Probability, vol. 104. Chapman \& Hall, London.

Rue, H., Martino, S. \& Chopin, N. (2009). Approximate Bayesian inference for latent Gaussian models by using integrated nested Laplace approximations. J R Stat Soc Series B Stat Methodol 71, 319-92.

SCHRÖDle, B. \& HELD, L. (2011a). A primer on disease mapping and ecological regression using INLA. Comput Stat 26, 241-258.

SCHRÖDle, B. \& Held, L. (2011b). Spatio-temporal disease mapping using INLA. Environmetrics 22, 725-734.

Schrödle, B., Held, L., Riebler, A. \& Danuser, J. (2011). Using integrated nested Laplace approximations for the evaluation of veterinary surveillance data from Switzerland: a case-study. $J$ $R$ Stat Soc Ser C Appl Stat 60, 261-279.

Simpson, D.P., Martins, T.G., Riebler, A., Fulglstad, G.A., Rue, H. \& Sørbye, S.H. (2014). Penalising model component complexity: A principled, practical approach to constructing priors. arXiv preprint arXiv:1403.4630 .

Sørbye, S.H. (2013). Tutorial: Scaling IGMRF-models in R-INLA. Department of Mathematics and Statistics, University of Tromsø .

Sørbye, S.H. \& Rue, H. (2014). Scaling intrinsic Gaussian Markov random field priors in spatial modelling. Spatial Statistics 8, 39-51.

Spiegelhalter, D.J., Best, N.G., Carlin, B.P. \& Linde, A.V.D. (2002). Bayesian measures of model complexity and fit. J R Stat Soc Series B Stat Methodol 64, 583-639.

TiERney, L. \& KADAne, J.B. (1986). Accurate approximations for posterior moments and marginal densities. J Am Stat Assoc 81, 82-86.

Ugarte, M.D., GoicoA, T., IBÁÑEZ, B. \& Militino, A.F. (2009). Evaluating the performance of spatiotemporal Bayesian models in disease mapping. Environmetrics 20, 647-665.

WAKEFIELD, J. (2007). Disease mapping and spatial regression with count data. Biostatistics 8, $158-83$.

Waller, L.A., Carlin, B.P., XiA, H. \& Gelfand, A.E. (1997). Hierarchical spatio-temporal mapping of disease rates. J Am Stat Assoc 92, 607-617. 


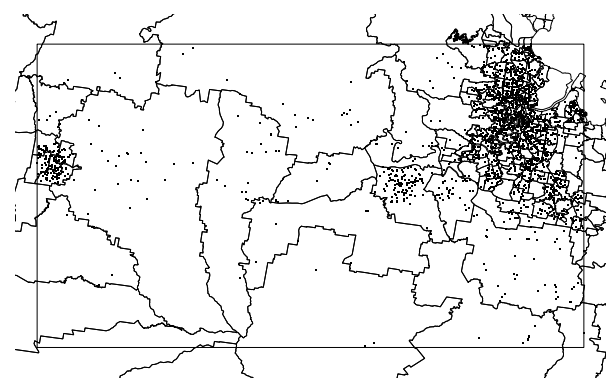

(a) Advanced breast cancer

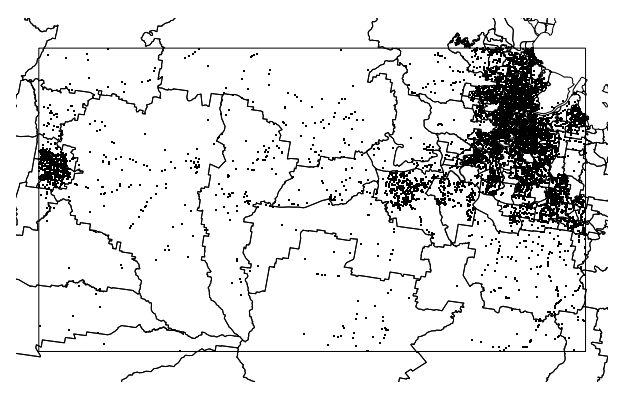

(b) Non-advanced breast cancer

Figure 1. Distribution of breast cancer cases from 1 January 1996 to 31 December 2009 in the study region which extends from Brisbane (upper right) to Toowoomba (middle left). The solid lines represent the SLA boundaries. (For reasons of confidentiality, the dots represent random locations within each SLA rather than actual addresses of cancer patients). 


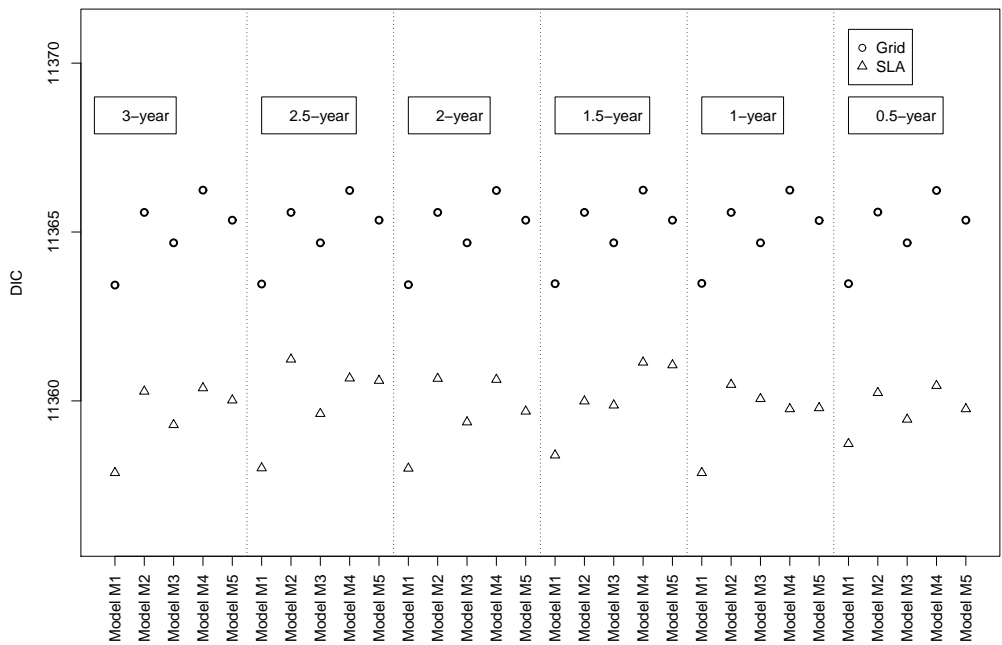

(a) DIC

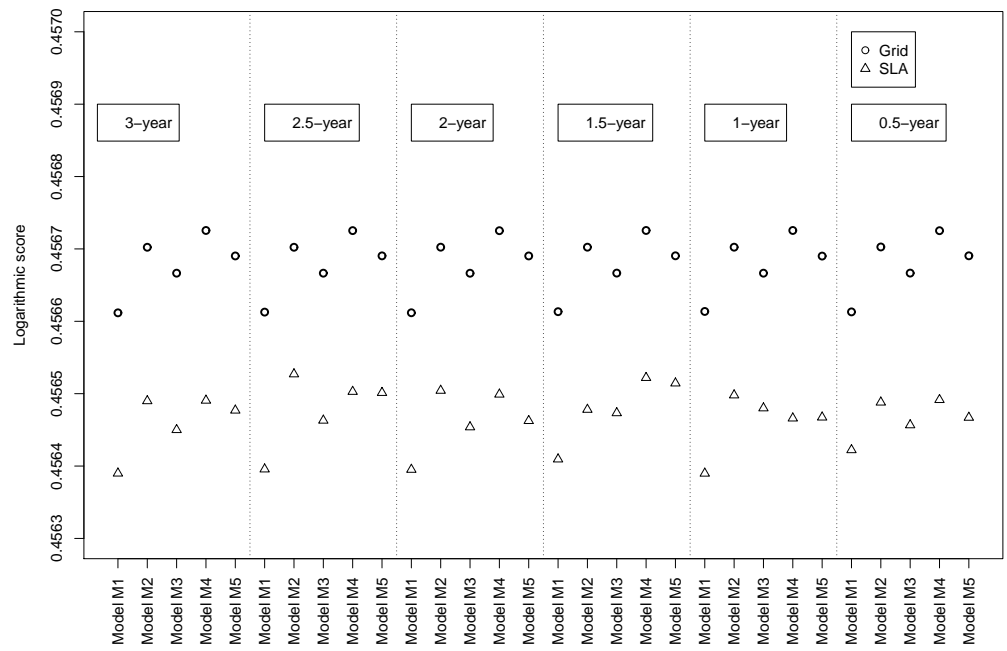

(b) LS

Figure 2. The DIC (top) and LS (bottom) for models M1 to M5 at all six levels of temporal aggregation and two levels of spatial aggregation. The results suggest that model M1 gave the best fit at all levels of aggregation. 


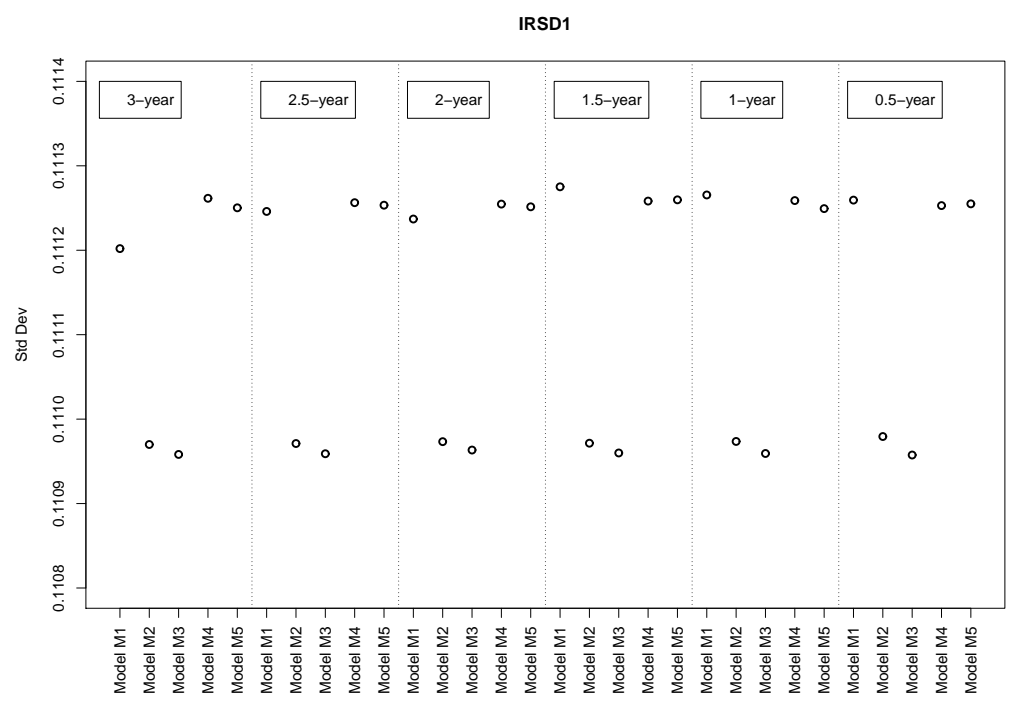

(a) Grid

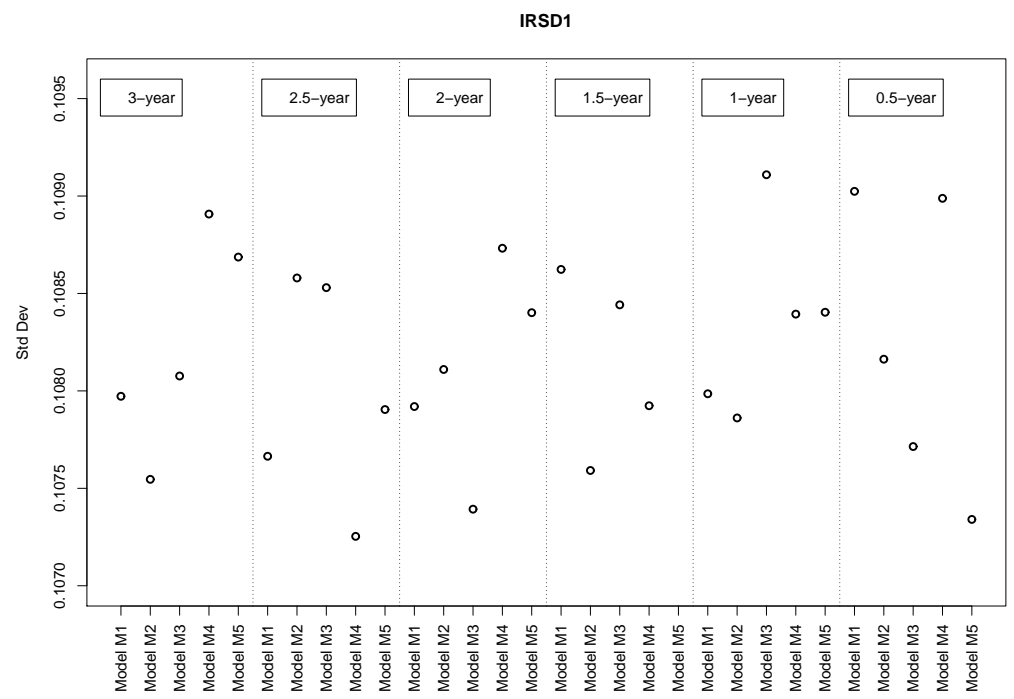

(b) SLA

Figure 3. Standard deviation of the posterior estimates of IRSD1 at all six levels of temporal aggregation at the grid level (top) and the SLA level (bottom). At each temporal aggregation level, from left to right is models M1 to M5. 


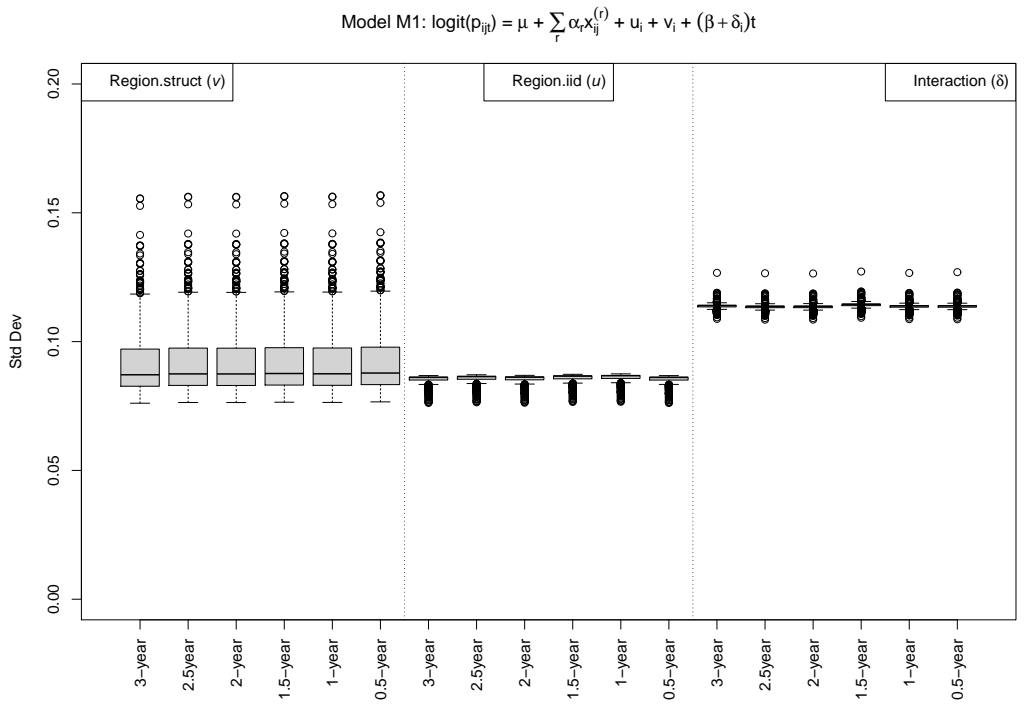

(a) Model M1

Figure 4. Box plots of the standard deviation of the random effects for model M1 at all six levels of temporal aggregation and the grid level. 'Region.struct' denotes the spatially structured random effects, 'Region.iid' denotes the spatially unstructured random effects, and 'Interaction' denotes the space-time interaction effects. 


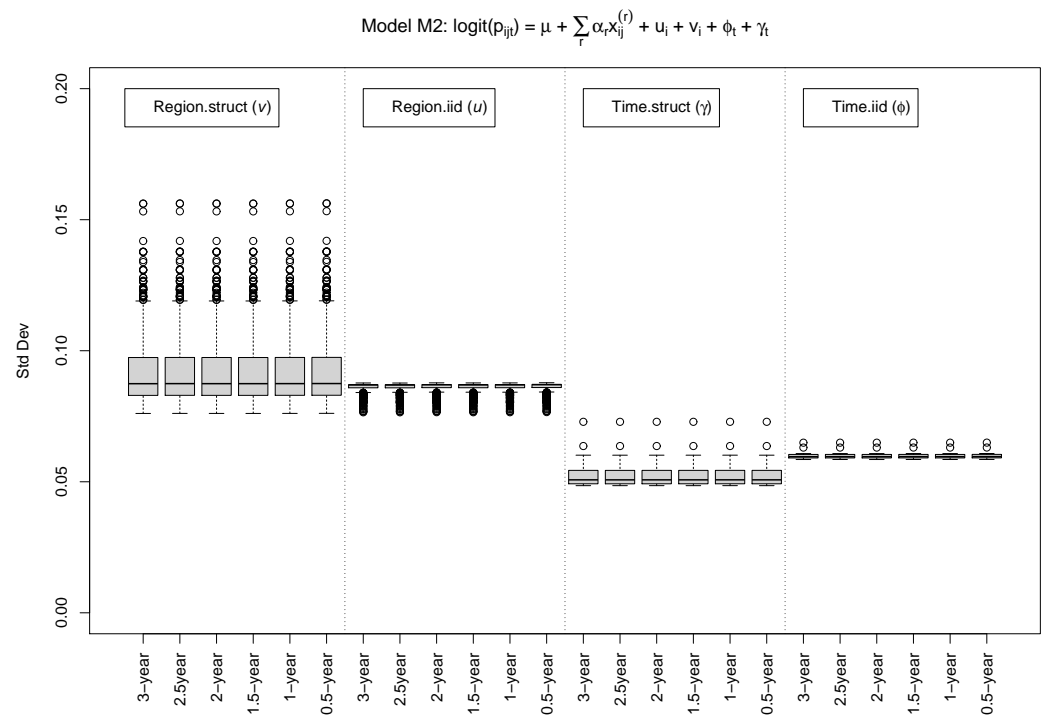

(a) Model M2

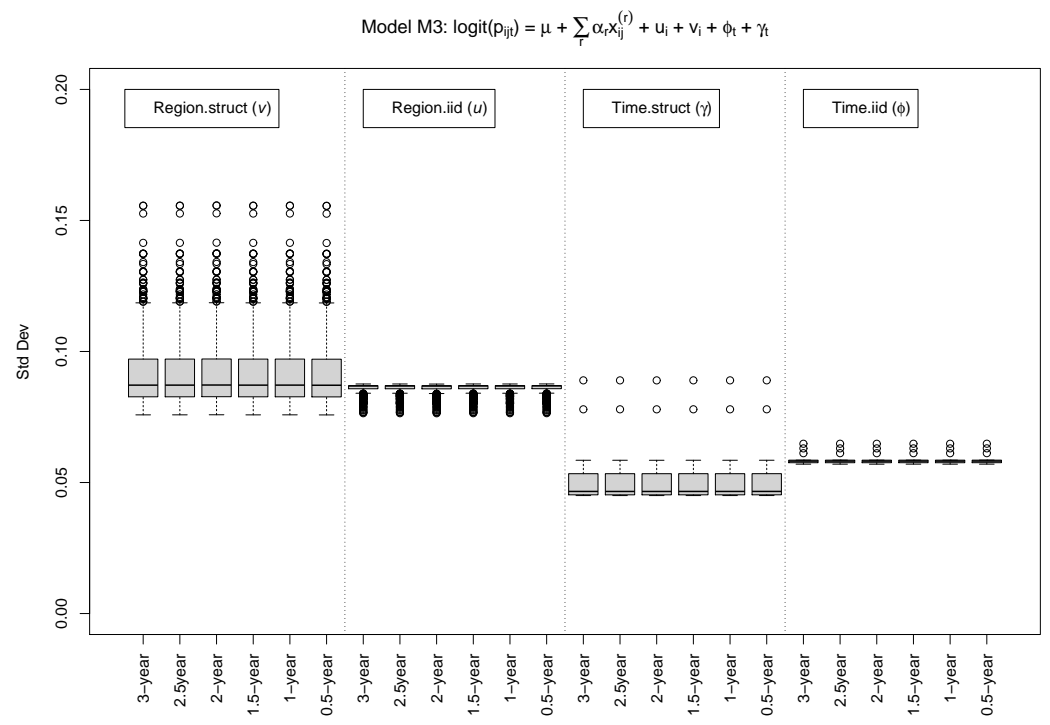

(b) Model M3

Figure 5. Box plots of the standard deviation of the random effects for models M2 (top) and M3 (bottom) at all six levels of temporal aggregation and the grid level. 'Region.struct' denotes the spatially structured random effects, 'Region.iid' denotes the spatially unstructured random effects, 'Time.struct' denotes the temporally structured random effects, and 'Time.iid' denotes the temporally unstructured random effects. 


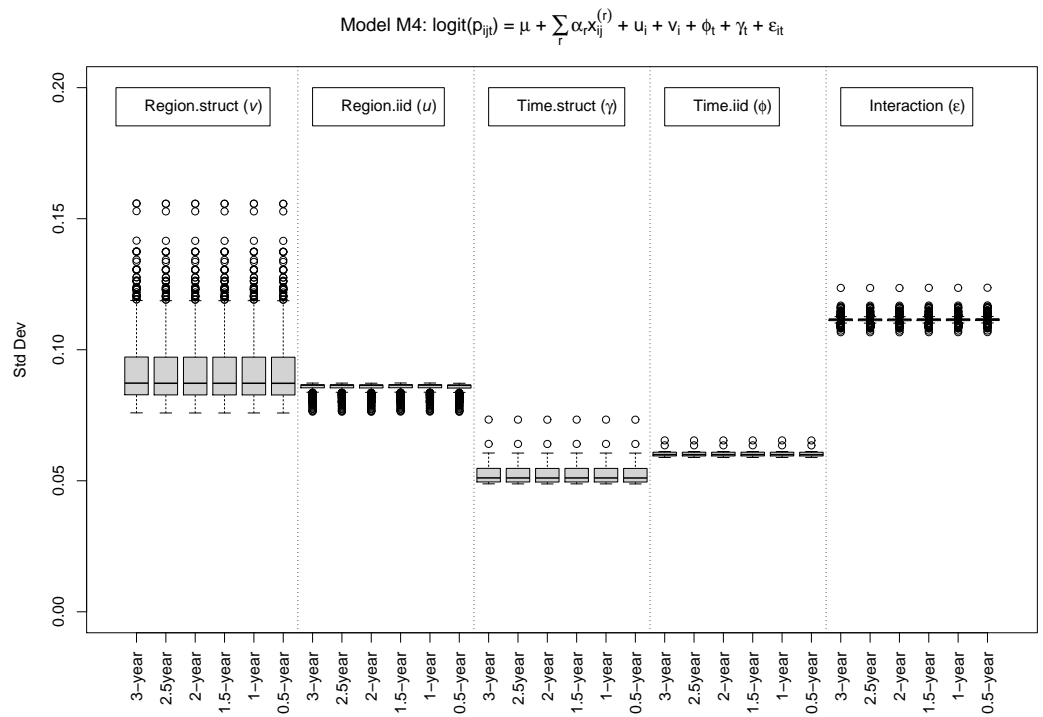

(a) Model M4

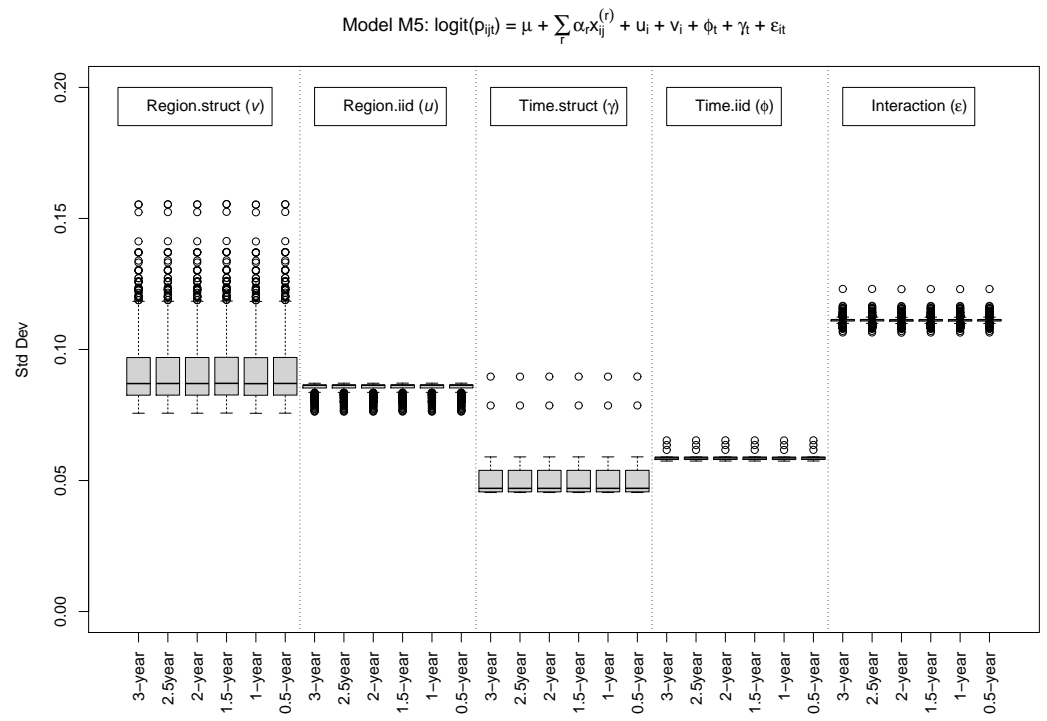

(b) Model M5

Figure 6. Box plots of the standard deviation of the random effects for models M4 (top) and M5 (bottom) at all six levels of temporal aggregation and the grid level. 'Region.struct' denotes the spatially structured random effects, 'Region.iid' denotes the spatially unstructured random effects, 'Time.struct' denotes the temporally structured random effects, 'Time.iid' denotes the temporally unstructured random effects, and 'Interaction' denotes the space-time interaction effects. 


\section{TABLE 1}

Description of models M1 to M5.

\begin{tabular}{lll}
\hline Model & Equation & Description \\
\hline M1 & $\operatorname{logit}\left(p_{i j t}\right)=\mu+\mathrm{IRSD}_{i j}+u_{i}+v_{i}+\left(\beta+\delta_{i}\right) t$ & $\begin{array}{l}\text { Classical parametric formulation } \\
\text { that assumes a linear time trend }\end{array}$ \\
\hline M2 & $\operatorname{logit}\left(p_{i j t}\right)=\mu+\mathrm{IRSD}_{i j}+u_{i}+v_{i}+\phi_{t}+\gamma_{t}$ & $\begin{array}{l}\text { Non-parametric formulation that } \\
\text { assumes a RW1 for the temporal } \\
\text { effects } \gamma\end{array}$ \\
\hline M3 & $\operatorname{logit}\left(p_{i j t}\right)=\mu+\mathrm{IRSD}_{i j}+u_{i}+v_{i}+\phi_{t}+\gamma_{t}$ & $\begin{array}{l}\text { Non-parametric formulation that } \\
\text { assumes a RW2 for the temporal } \\
\text { effects } \gamma\end{array}$ \\
\hline M4 & $\operatorname{logit}\left(p_{i j t}\right)=\mu+\mathrm{IRSD}_{i j}+u_{i}+v_{i}+\phi_{t}+\gamma_{t}+\epsilon_{i t}$ & $\begin{array}{l}\text { Non-parametric formulation with } \\
\text { space-time interaction effect } \epsilon \text { that } \\
\text { assumes a RW1 for the temporal } \\
\text { effects } \gamma\end{array}$ \\
& & $\begin{array}{l}\text { Non-parametric formulation with } \\
\text { space-time interaction effect } \epsilon \text { that } \\
\text { assumes a RW2 for the temporal } \\
\text { effects } \gamma\end{array}$ \\
\hline M5 & $\operatorname{logit}\left(p_{i j t}\right)=\mu+\mathrm{IRSD}_{i j}+u_{i}+v_{i}+\phi_{t}+\gamma_{t}+\epsilon_{i t}$ \\
& & \\
\hline
\end{tabular}


TABLES

TABLE 2

63 combinations of the covariates.

\begin{tabular}{|c|c|c|c|c|c|c|c|}
\hline & Model & AGE & OCCUP & MARITAL & INDIG & ARIA & IRSD \\
\hline 6 covariates & 1 & $*$ & $*$ & $*$ & $*$ & $*$ & $*$ \\
\hline \multirow{6}{*}{5 covariates } & 1 & $*$ & * & * & * & * & \\
\hline & 2 & * & * & * & * & & * \\
\hline & 3 & * & $*$ & $*$ & & * & * \\
\hline & 4 & * & $*$ & & * & * & * \\
\hline & 5 & * & & * & * & * & * \\
\hline & 6 & & $*$ & * & * & * & * \\
\hline \multirow{15}{*}{4 covariates } & 1 & * & * & * & * & & \\
\hline & 2 & * & * & * & & * & \\
\hline & 3 & $*$ & $*$ & $*$ & & & * \\
\hline & 4 & * & * & & * & * & \\
\hline & 5 & * & * & & * & & * \\
\hline & 6 & * & $*$ & & & * & * \\
\hline & 7 & * & & * & * & * & \\
\hline & 8 & * & & * & * & & * \\
\hline & 9 & * & & $*$ & & * & * \\
\hline & 10 & * & & & * & * & * \\
\hline & 11 & & * & * & * & * & \\
\hline & 12 & & * & * & * & & * \\
\hline & 13 & & $*$ & $*$ & & * & * \\
\hline & 14 & & $*$ & & * & * & * \\
\hline & 15 & & & * & $*$ & * & * \\
\hline \multirow{20}{*}{3 covariates } & 1 & * & * & * & & & \\
\hline & 2 & * & * & & * & & \\
\hline & 3 & * & * & & & * & \\
\hline & 4 & $*$ & $*$ & & & & * \\
\hline & 5 & * & & $*$ & * & & \\
\hline & 6 & * & & * & & * & \\
\hline & 7 & $*$ & & $*$ & & & $*$ \\
\hline & 8 & * & & & * & * & \\
\hline & 9 & * & & & * & & * \\
\hline & 10 & * & & & & * & * \\
\hline & 11 & & $*$ & $*$ & * & & \\
\hline & 12 & & $*$ & $*$ & & * & \\
\hline & 13 & & * & * & & & * \\
\hline & 14 & & * & & * & * & \\
\hline & 15 & & * & & $*$ & & $*$ \\
\hline & 16 & & * & & & * & * \\
\hline & 17 & & & * & * & * & \\
\hline & 18 & & & * & * & & * \\
\hline & 19 & & & $*$ & & * & * \\
\hline & 20 & & & & $*$ & * & * \\
\hline \multirow{15}{*}{2 covariates } & 1 & $*$ & * & & & & \\
\hline & 2 & * & & * & & & \\
\hline & 3 & $*$ & & & $*$ & & \\
\hline & 4 & * & & & & $*$ & \\
\hline & 5 & $*$ & & & & & $*$ \\
\hline & 6 & & $*$ & $*$ & & & \\
\hline & 7 & & $*$ & & * & & \\
\hline & 8 & & $*$ & & & $*$ & \\
\hline & 9 & & $*$ & & & & $*$ \\
\hline & 10 & & & * & * & & \\
\hline & 11 & & & $*$ & & $*$ & \\
\hline & 12 & & & $*$ & & & $*$ \\
\hline & 13 & & & & * & * & \\
\hline & 14 & & & & $*$ & & $*$ \\
\hline & 15 & & & & & $*$ & $*$ \\
\hline \multirow{6}{*}{1 covariate } & 1 & $*$ & & & & & \\
\hline & 2 & & $*$ & & & & \\
\hline & 3 & & & $*$ & & & \\
\hline & 4 & & & & $*$ & & \\
\hline & 5 & & & & & $*$ & \\
\hline & 6 & & & & & & $*^{\mathrm{a}}$ \\
\hline
\end{tabular}

${ }^{\text {a }}$ This model has the smallest DIC value and parameter estimates that do not contain zero in the $90 \%$ credible interval. 
TABLE 3

Posterior probability $\left(\mathrm{P}\left(\alpha_{p}<0\right)\right)$ of the regression parameters for models M1 to M5 at all six levels of temporal aggregation at the grid and SLA levels.



